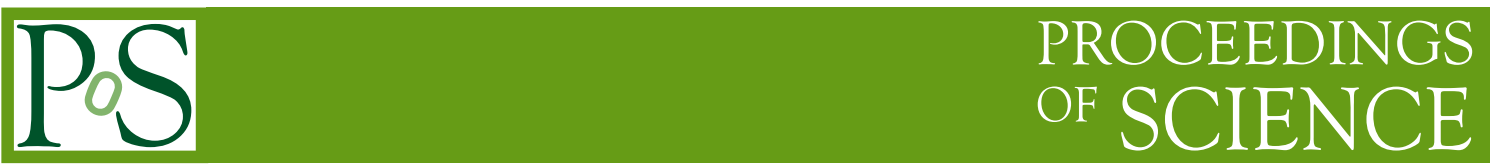

\title{
Soft X-ray excess emission in NLS1 galaxies
}

\author{
Main Pal* \\ Astronomy \& Astrophysics Division, Physical Research Laboratory, Ahmedabad, 380009; \\ E-mail: mainpaleprl.res.in
}

\begin{abstract}
A long-standing puzzle of the origin of soft X-ray excess in Seyfert 1 galaxies remains a focus in the research area of active galactic nuclei. That this was considered the origin of soft excess could be thermal emission from the inner accretion disk. This component has been explained with a temperature $0.1-0.3 \mathrm{keV}$ range of the masses $10^{6-9} M_{\odot}$ of supermassive black hole and a range of accretion rates. Such a hot disk is not expected in these AGNs and rarely it can attain the temperature of about $100 \mathrm{eV}$ for low mass and high accretion rate. We tested other two competing possibilities-blurred reflection from partially ionized accretion disk and the cool Comptonization of seed photons from the accretion disk. We applied these two models using XMM-Newton and NuSTAR observations of two NLS1 Mrk 766 and II Zw 177. What we found that both models describe equally good to the broadband spectrum for both AGNs. Time resolved spectroscopy sheds some light on the origin and likely related to the blurred reflection when geometry (size or height) of the X-ray corona changes.
\end{abstract}

Revisiting narrow-line Seyfert 1 galaxies and their place in the Universe - NLS1 Padova

9-13 April 2018

Padova Botanical Garden, Italy

${ }^{*}$ Speaker. 


\section{Introduction}

Active galactic nuclei (AGNs) exhibit complex spectral energy distribution (SED) and are found to be highly variable. These AGNs are broadly classified based on their optical properties (Osterbrock \& Pogge 1985). There are two major components of such classification and those are known as the broad line Seyfert 1 (BLS1) galaxy and the narrow-line Seyfert 1 (NLS1) galaxy. NLS1 galaxies show peculiar features which separate them from BLS1. Some of the most important features are the full width at half maximum, the strength of Fe II line, the ratio of $\mathrm{OIII} / \mathrm{H}_{\beta}$ observed in the optical spectra (Osterbrock \& Pogge 1985). The full width at half maximum for NLS1 is considered less than $2000 \mathrm{~km} \mathrm{~s}^{-1}$. The strength of Fe II line is stronger than that of found in BLS1 and the ratio for $\left[\mathrm{O} \mathrm{III]} / \mathrm{H}_{\beta}\right.$ is found to be less than or equal to 3. Similarly, the X-ray spectrum of NLS1 also shows differences compared to the BLS1. The stronger soft X-ray X-ray, steeper 2-10 keV power-law index and extreme X-ray variability are some of the main properties of these NLS1 AGN over BLS1 (Leighly 1999). These objects show SED over the entire electromagnetic spectrum of radiation emitted from various components such as accretion disc, jet, corona, torus e.g., Romano et al.(2004). The X-ray to UV/Optical spectrum of radio-quiet Seyfert 1 galaxies, including NLS1 consists of main spectral components - power-law continuum, Compton reflection hump, iron $\mathrm{K}$ emission line, the big blue bump and soft X-ray excess over power-law continuum.

The power-law emission is expected due to the Compton up-scattering of the UV/Optical emission from the accretion disk in the hot electron plasma (Haardt \& Maraschi 1991). The Compton scattering hump is normally thought to arise through the back-scattering of high energy photons from the optically thick material such as putative torus (Lightman \& White 1988). The iron K emission line is generated as a result of photoabsorption followed by the fluorescence emission. Sometimes these two spectral components (Compton reflection hump and iron K emission line) are termed as the reflection components. The big blue bump is the crucial component of these sources and this is emanated from the accretion disk (Koratkar \& Blaes 1999). The soft excess is still under debate and no definite solution has been found to explain this component (Crummy et al 2006, Done et al 2012, Gierlinski \& Done 2004, Kawaguchi 2003).

Sometimes, the Wien's tail of disk blackbody can partly contribute in the soft X-ray excess emission. The temperature of the standard accretion disk (Shakura \& Sunyaev 1973) at a distance $r$ can be estimated by following expression

$$
T(r) \sim 6.3 \times 10^{5}\left(\frac{\dot{M}}{M_{8}}\right)^{1 / 4}\left(\frac{r}{R_{S}}\right)^{-3 / 4}
$$

where $\dot{M}, M_{8}, \mathrm{R}_{S}$ are the accretion rate in units of Eddington rate, mass of the black hole in units of $10^{8} M_{\odot}$ and the Schwarzschild radius, respectively. According to this function, the temperature of the disk varies inversely proportional to the three fourths power of radius of the disk from the centre of black hole. The inner disk becomes hotter and hotter than the outer disk. At a given radius of disk, the disk is much hotter for low mass of the black hole and high accretion rate objects such as NLS1. It has been found that the temperature of the disk is in the range 0.1-0.3 keV for a large number of Seyfert 1 galaxies (Leighly 1999). The constancy of temperature of the inner accretion disk indicates that the origin of the soft excess may be related to the atomic physics in this band. There were possible models related to atomic physics to explain this component-the blurred 
reflection from the partially ionized accretion disk (Ross \& Fabian 2005) and smeared of absorption features associated to the partially ionized moving absorber close to the SMBH (Gierlinski \& Done 2004). The absorption origin of the soft excess has been ruled out due to the requirement of very high velocity of absorber (Schurch \& Done 2008). In addition, there have been another possible explanation for the soft excess caused by the cool Comptonization (Done et al. 2012). Therefore, at present, there are two competing models- blurred reflection and cool Comptonization.
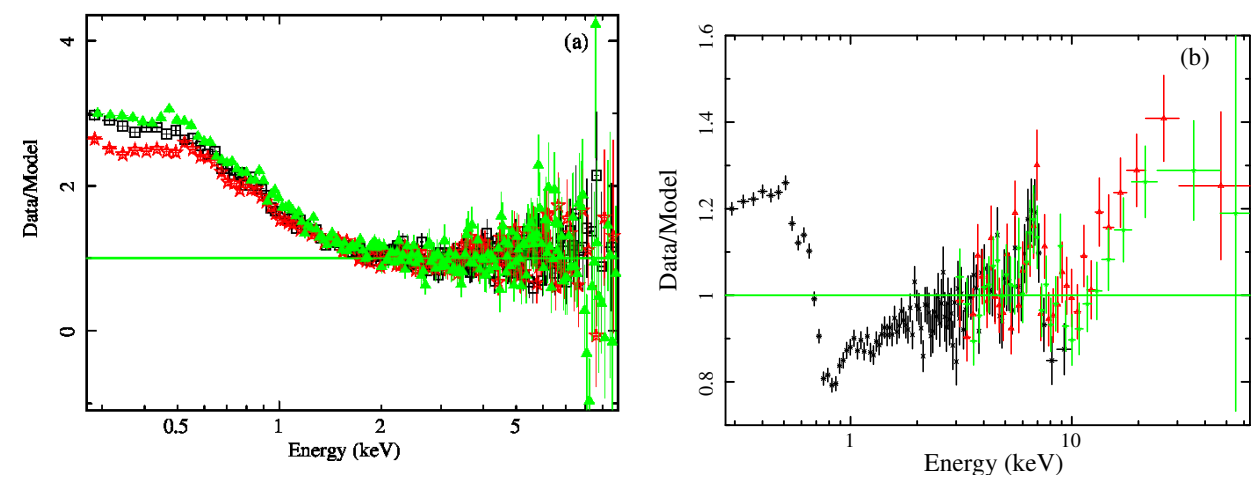

Figure 1: (a) II Zw 177 : Residuals (Data/Model) are shown for the 2-10 keV fit by absorbed powerlaw and its extrapolation down to $0.3 \mathrm{keV}$ using EPIC-pn, MOS 1 and MOS 2 instruments. Clearly an excess is visible below $2 \mathrm{keV}$. MOS 1 shows some difference due to calibration issues and hence the data below $0.5 \mathrm{keV}$ was discarded from the analysis. (b) Mrk 766 : Residuals in Data/Model ratio are displayed for absorbed powerlaw fit in the 3-10 keV band and the the fit was extrapolated down to $0.3 \mathrm{keV}$ using EPIN-pn data and upto $60 \mathrm{keV}$ using the NuSTAR modules FPMA/FPMB. The presence of soft excess below $1 \mathrm{keV}$, narrow and broad iron line near $6 \mathrm{keV}$ and the Compton reflection hump above $10 \mathrm{keV}$ are very prominent.

The SED of NLS1 is largely composed of mixture of absorption components such as warm/neutral absorbers including all above spectral components. To understand the origin of soft X-ray excess, one has to remove the effects of absorption which can modify the X-ray emission in a complex way. In the spectral modeling, the reflection and Comptonization models can describe equally well to the soft X-ray excess as well as the broadband X-ray emission (Pal et al. 2016, Pal, M \& Dewangan G. C. 2013). In such cases, the variability techniques can play an important role to disentangle the ambiguity between models (Pal et al 2016). Also the time information between various energy bands can also provide important clues about the different physical processes (Pal et al 2018, Zoghbi et al. 2010, Zoghbi et al. 2011).

\section{Observational details}

We analysed observations obtained from XMM-Newton and NUSTAR observatories for two NLS1 II Zw 177 and Mrk 766. We followed standard procedure for data reduction to get science products. For II Zw 177, we used 2012 observation using EPIC-pn, MOS 1 and MOS 2 in the 0.3-10 keV band with a total exposure of about $130 \mathrm{ks}$. We also used Optical Monitor observations simultaneous to the X-ray during 2012 epoch (see Pal, M. et al. 2016). On the other hand, we used simultaneous XMM-Newton and NUSTAR 2015 July observations for Mrk 766 for the first time. The data reduction procedure for NuSTAR observations was followed as described in NuSTAR Data Analysis Software Users guide. The exposure time of both XMM-Newton and NuSTAR observations 
were about $30 \mathrm{ks}$. We used the datasets of EPIC-pn and both onboard modules FPMA and FPMB in the $0.3-60 \mathrm{keV}$ band.
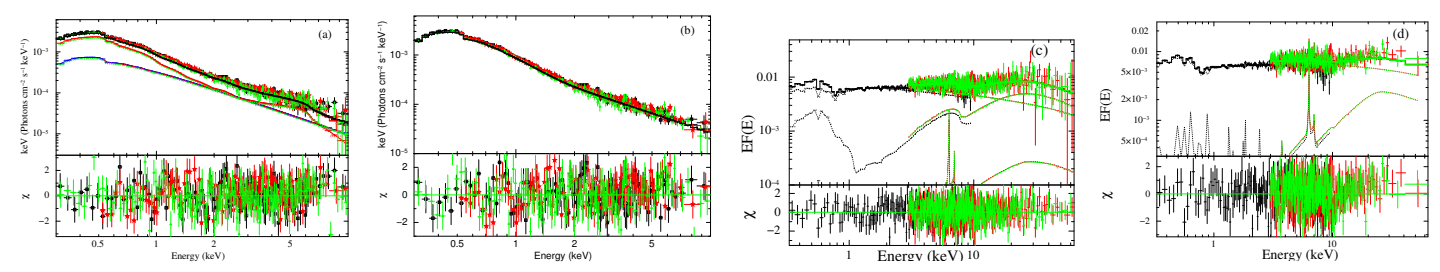

Figure 2: Upper panels: Broad-band spectral fitting to the data-sets using blurred reflection and cool Comptonization models for II Zw 177 : (a) blurred reflection+powerlaw, (b) Comptonization with two Comptoning component - optically thick, cool plasma and optically thin, hot plasma; Mrk 766 (c) blurred reflection+powerlaw + distant reflection, (d) Comptonization with two Comptoning component - optically thick, cool plasma and optically thin, hot plasma plus distant reflection. Lower panels : residuals are shown in units of $\sigma$.

\section{Results and discussion}

We analysed datasets for two bright, highly variable NLS1 II Zw 177 and Mrk 766 galaxies. II Zw 177 shows strong soft X-ray excess below $2 \mathrm{keV}$ as shown in figure 1 (a) while Mrk 766 observation exhibits the soft excess below $1 \mathrm{keV}$ (see figure 1 (b)). Presence of strong soft excess in both AGN makes them good targets to understand the origin of the soft X-ray excess. Since the blackbody temperature observed for soft excess are found to be in the $0.1-0.3 \mathrm{keV}$ range for a range of mass of SMBH and accretion rate, such a constant nature of this component hinted some atomic physics origin. The blurred reflection from partially ionized accretion disk has been likely one of the best choices for explaining the soft excess (Pal et al. 2016, Fabian et al 2002). Another possible origin is related to the inner disk itself, where a different type of plasma of low temperature and optically thick in nature lies (Done et al. 2012). We applied both the models to describe the soft excess and fitted the broadband X-ray for both cases ( 0.3-10 keV and 0.3-60 keV for II Zw 177 and Mrk 766, respectively).

In case of II $\mathrm{Zw} 177$, we found that the emissivity index was observed to be $q \sim 4$ which is resulted due to the strong light bending effect in the reflection scenario. Similarly the cool Comptonizing plasma ( $\mathrm{kT}_{e} \sim 0.2 \mathrm{keV}, \tau \sim 20$ ) is capable to explain the soft excess equally good statistically and physically. We therefore found that the blurred reflection and the cool Comptonization models describe equally well to the data in both cases (see figure 2 (a) and 2 (b)) and the spectral fitting procedure is unable to remove the degeneracy. Time resolved spectroscopy was able to provide a way to distinguish between these two models. We divided the X-ray light curve of EPIC-pn into six time-segment of about $20 \mathrm{ks}$ where each segment consists of one simultaneous exposure of $U$ band. We derived the spectral products of for each time-segment of Epic-pn spectrum in the 0.3-10 keV band. We fitted a phenomenological composite model using a blackbody and a simple power law in the $0.3-10 \mathrm{keV}$ band.

The results are shown in the left panel of figure 3. Interestingly, we found that the soft excess flux and power-law flux are strongly correlated which is not expected in the simple light bending model under lamppost geometry. We also checked the effect of flares presence in the X-ray light 

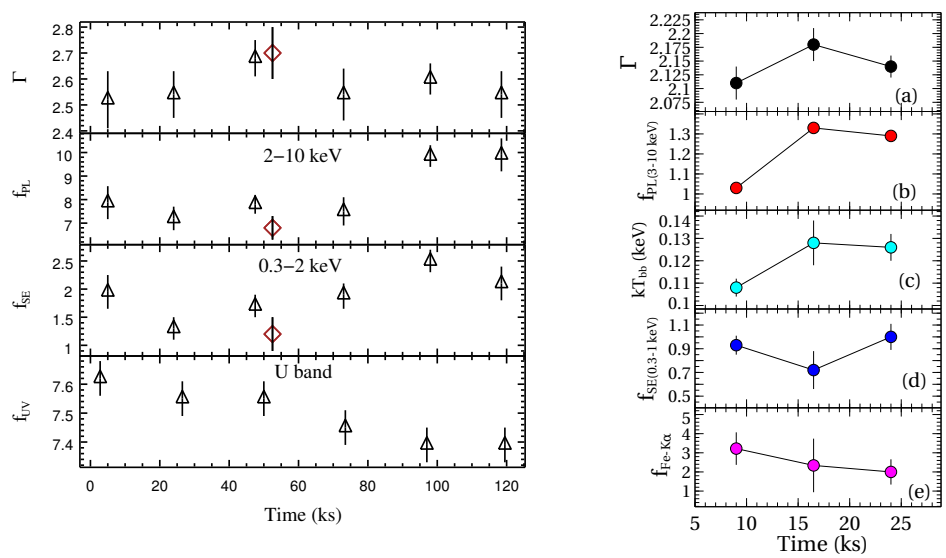

Figure 3: Time-resolved spectroscopic results using simple blackbody for soft excess and powerlaw for high energy X-ray emission: Left panel: II Zw 177 - the spectral parameters are shown for II Zw 177- powerlaw photon index, powerlaw flux, soft excess flux and UV flux from top to bottom panels, respectively. Mrk 766 the photon index, 3-10 keV power-law flux, innder disk temperature, soft excess flux and broad iron flux are shown from top to bottom, respectively. Units of X-ray fluxes and UV flux density are $10^{-11} \mathrm{ergs} \mathrm{s}^{-1} \mathrm{~cm}^{-2}$ and $10^{-11} \mathrm{ergs} \mathrm{s}^{-1} \mathrm{~cm}^{-2}$ Angstrom $^{-1}$, respectively.

curve, whether flare affects the results or not. After excluding the flare from X-ray light curve as shown by red diamond notation in the left panel of figure 3, we found a stronger correlation between power-law flux and soft excess flux. At the same time the power-law photon index does not change with time suggesting no significant variation in power-law flux and soft excess flux due to Comptonization. Moreover, the UV emission is not correlated with any component such as photon index, fluxes of power-law and soft excess. The variations observed in power-law and soft excess fluxes infer that the changes in the geometry of the corona are likely playing an important role in the frame of reflection. Also, the observed secular decline could be resulted due to the changes in accretion rate which normally shows fluctuations on thousands of years time-scale.

In the analysis of Mrk 766, we used blackbody, blurred reflection and cool Comptonization models to describe the soft excess. We observed blackbody temperature of about $90 \mathrm{eV}$, which is too hot to explain the soft excess physically. The soft excess was fitted using reflection and Comptonization models, as shown in figure 2 (c) and 2 (d), respectively. Both blurred reflection ( $q \sim 4, \mathrm{R}_{\text {in }} \sim 2 \mathrm{r}_{\mathrm{g}}$, where $\mathrm{r}_{g}$ is gravitational radius) the cool Comptonizing plasma $\left(\mathrm{kT}_{e} \sim 0.12\right.$ $\mathrm{keV}, \tau \geq 66)$ are able to explain the soft excess. This spectral modeling provided an equally good description and we were unable to disentangle such a degeneracy. The entire EPIC-pn light curve for time-resolved spectroscopy was split into three time-segments. The spectrum of each segment was fitted using simple power law and blackbody models along with a broad Gaussian for iron line modified by a warm absorber component in the $0.3-10 \mathrm{keV}$ band.

The fit revealed interesting results which are shown in the right panel of figure 3 . The photon index, power-law flux and blackbody temperature seem correlated while the soft excess flux and broad iron $\mathrm{K}$ emission line flux appear unchanged. This suggests a complex picture in the vicinity of the SMBH. This variation in photon index hints the presence of Comptonization while the correlation powerlaw flux and blackbody temperature indicates complicated scenario caused by intense 
radiation incidence. Due to intense irradiation, the temperature of blackbody is increased due to reprocessing of incident radiation. At the same time, the flux of blackbody does not vary (see right panel of figure 4) and this may be a scenario where most of the flux of soft excess is unable to escape from the interior region and enters in the blackhole. Thus, both Comptonization as well as strong light bending may be likely playing an important role to explain the soft excess.

\section{Acknowledgements}

This conference has been organized with the support of the Department of Physics and Astronomy "Galileo Galilei", the University of Padova, the National Institute of Astrophysics INAF, the Padova Planetarium, and the RadioNet consortium. RadioNet has received funding from the European Union's Horizon 2020 research and innovation programme under grant agreement No 730562. MP thanks the Physical Research Laboratory, Ahmedabad for financial support towards travel and also acknowledges Prof. Sachindra Naik for his help making it a successful trip . MP also thanks to Prof. Chris Done and Prof. Luigi Gallo for their useful discussion and comments.

\section{References}

[1] Haardt, F., \& Maraschi, L. 1991, ApJ, 380, L51

[2] Pal, M., Dewangan, G. C., Misra, R., \& Pawar, P. K. 2016, MNRAS, 457, 875

[3] Osterbrock, D. E., \& Pogge, R. W. 1985, ApJ, 297, 166

[4] Leighly, K. M. 1999, ApJS, 125, 317

[5] Pounds, K. A., Done, C., \& Osborne, J. P. 1995, MNRAS, 277, L5

[6] Crummy, J., Fabian, A. C., Gallo, L., \& Ross, R. R. 2006, MNRAS, 365, 1067

[7] Done, C., Davis, S. W., Jin, C., Blaes, O., \& Ward, M. 2012, MNRAS, 420, 1848

[8] Pal, M., \& Naik, S. 2018, MNRAS, 474, 5351

[9] Lightman, A. P., \& White, T. R. 1988, ApJ, 335, 57

[10] Dewangan, G. C., Griffiths, R. E., Dasgupta, S., \& Rao, A. R. 2007, ApJ, 671, 1284

[11] Pal, M., Dewangan, G. C., Kembhavi, A. K., Misra, R., \& Naik, S. 2018, MNRAS, 473, 3584

[12] Schurch, N. J., \& Done, C. 2008, MNRAS, 386, L1

[13] Koratkar, A., \& Blaes, O. 1999, PASP, 111, 1

[14] Singh, K. P., Garmire, G. P., \& Nousek, J. 1985, ApJ, 297, 633

[15] Shakura, N. I., \& Sunyaev, R. A. 1973, A\&A, 24, 337

[16] Pal, M., \& Dewangan, G. C. 2013, MNRAS, 435, 1287

[17] Romano, P., Mathur, S., Turner, T. J., et al. 2004, ApJ, 602, 635

[18] Gierliński, M., \& Done, C. 2004, MNRAS, 349, L7

[19] Ross, R. R., \& Fabian, A. C. 2005, MNRAS, 358, 211

[20] Zoghbi, A., \& Fabian, A. C. 2011, MNRAS, 418, 2642

[21] Zoghbi, A., Fabian, A. C., Uttley, P., et al. 2010, MNRAS, 401, 2419

[22] Kawaguchi, T. 2003, ApJ, 593, 69 